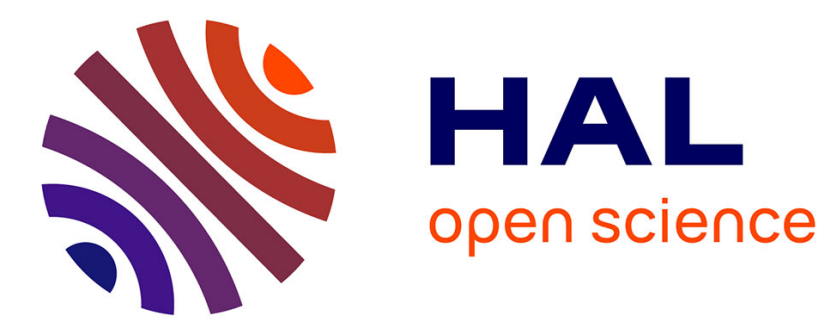

\title{
Modifier Genes in Cystic Fibrosis-related Liver Disease
}

Dominique Debray, Harriet Corvol, Chantal Housset

\section{To cite this version:}

Dominique Debray, Harriet Corvol, Chantal Housset. Modifier Genes in Cystic Fibrosisrelated Liver Disease. Current Opinion in Gastroenterology, 2019, 35 (2), pp.88-92. 10.1097/MOG.0000000000000508 . hal-02178958

\section{HAL Id: hal-02178958 \\ https://hal.sorbonne-universite.fr/hal-02178958}

Submitted on 10 Jul 2019

HAL is a multi-disciplinary open access archive for the deposit and dissemination of scientific research documents, whether they are published or not. The documents may come from teaching and research institutions in France or abroad, or from public or private research centers.
L'archive ouverte pluridisciplinaire HAL, est destinée au dépôt et à la diffusion de documents scientifiques de niveau recherche, publiés ou non, émanant des établissements d'enseignement et de recherche français ou étrangers, des laboratoires publics ou privés. 


\section{Modifier Genes in Cystic Fibrosis-related Liver Disease}

Dominique Debray, ${ }^{1,2}$ Harriet Corvol, ${ }^{1,3}$ Chantal Housset. ${ }^{1,4}$

${ }^{1}$ Sorbonne Université, INSERM, Centre de Recherche Saint-Antoine (CRSA), Paris, France ;

${ }^{2}$ Assistance Publique-Hôpitaux de Paris (AP-HP) - Université Paris V, Hôpital Necker Enfants Malades, Pediatric Hepatology Unit, Reference Centre for Biliary Atresia and Genetic Cholestatic Diseases (CRMR, AVB-CG), Paris, France ;

${ }^{3}$ AP-HP, Hôpital Trousseau, Pneumologie Pédiatrique, Paris, France ;

${ }^{4}$ AP-HP, Hôpital Saint-Antoine, Reference Centre for Inflammatory Biliary Diseases and Autoimmune Hepatitis (CRMR, MIVB-H), Department of Hepatology, Paris, France

Corresponding author:

Dominique Debray, MD, PhD

Pediatric hepatology Unit

Department of Gastroenterology, Hepatology and Nutrition.

Hôpital Necker-Enfants Malades, 149 rue de Sèvres, 75015 Paris, France

Phone : + 33144492588

E-mail: dominique.debray@aphp.fr

Abbreviations : ACE, angiotensin I converting enzyme; CF, cystic fibrosis; CFLD, CF-related liver disease; CFTR, cystic fibrosis transmembrane conductance regulator; GSTP1, glutathione S-transferase P1; MBL2, mannose binding lectin 2; NF-кB, nuclear factor kappa-light-chainenhancer of activated B cells; PAI-1, plasminogen activator inhibitor-1; TGFB1, transforming growth factor beta 1; TIMP-1, tissue inhibitor of metalloproteinase-1, TLR, toll-like receptor.

Aknowledgments : none

Financial support and sponsorship: This work was supported by the Cystic Fibrosis Patients Association Vaincre la Mucoviscidose, Paris, France

Conflicts of interest: none 


\begin{abstract}
Purpose of review: Cystic fibrosis (CF; OMIM 219700) is caused by variations in the cystic fibrosis transmembrane conductance regulator (CFTR) gene. CF- related liver disease (CFLD) affects approximately one third of patients with CF, but the severity of CFLD is highly variable. This review provides the latest knowledge in the pathophysiology and CF genetic modifier research in CFLD.

Recent findings: So far, the only modifier gene validated in CFLD is SERPINA1 (alpha-1antitrypsin) $\mathrm{Z}$ allele. Recent studies support the view that cholangiopathy arising in $\mathrm{CF}$, is the result of an ill-adapted innate immune response to endotoxins coming from the intestine and triggering a pro-inflammatory response.

Summary: The pathophysiology of liver disease remains uncertain and so far, no therapy has proven effective to prevent the progression of CFLD. A better understanding of the pathophysiology and the effect of environmental and non-CFTR genetic influences in the context of CFLD development would help improve management and develop new drug therapies.
\end{abstract}

Keywords: cystic fibrosis, cholangiopathy, innate immunity, Serpina1

Word counts: Abstract : 162 words; Text : 2470 words 


\section{Introduction}

Cystic fibrosis (CF; OMIM 219700) is the most common autosomal recessive disease among Caucasians. CF is caused by variations in the cystic fibrosis transmembrane conductance regulator $(C F T R)$ gene. Estimates of incidence are of approximately 1 for every 3,000 live births worldwide ${ }^{1}$. The secretory defect in CF, leads to a decrease in the luminal hydration of ducts, physicochemical abnormalities of secretions and duct obstruction. It is a multiorgan disease affecting mostly the lungs, pancreas, sweat glands, and the Wolffian ducts in males. Other clinically relevant manifestations include intestinal obstruction in the neonatal period (meconium ileus) or later in life (distal intestinal obstructive syndrome), and hepatobiliary disease. The median age of survival has increased overtime to exceed 40 years, as a result of neonatal screening and improved management, particularly of lung disease. With the recent development of CFTR-directed therapies that correct the function of the defective protein, life expectancy of individuals with CF should continue to increase ${ }^{2}$.

The clinical phenotype in CF shows considerable variability. Both the number of affected organs and the extent of damage within a specific organ vary substantially between patients with CF. This is particularly true as far as the lung and liver disorders are concerned ${ }^{3,4}$.

Liver disease has emerged as a significant contributor to the morbidity and mortality of patients with CF. In 2014, CF-related liver disease (CFLD) was the third most common cause of mortality after cardiorespiratory and transplant-related causes, responsible for approximately $2.8 \%$ of deaths ${ }^{5}$. Focal biliary cirrhosis is the classical histological lesion of CFLD. It has been ascribed to CFTR defect in bile duct epithelial cells (i.e, cholangiocytes), which would result in biliary obstruction by inspissated secretions and progressive periportal fibrosis. Focal biliary cirrhosis has been reported in up to $70 \%$ in autopsy studies of CF patients ${ }^{6}$. However, the progression from focal biliary fibrosis to multilobular cirrhosis, which most often manifests as complications of portal hypertension by the end of the second decade of life, occurs in a 
minority of patients (5 to $10 \%)^{4,7-13}$. In the largest series of CF patients with cirrhosis and portal hypertension (561 in total), $90 \%$ were diagnosed by 18 years of age with a mean age at diagnosis of 10 years ${ }^{8}$. The progression to cirrhosis and portal hypertension remains unpredictable in the absence of sensitive biomarkers ${ }^{13}$. The other biliary manifestations of CF, including large-duct sclerosing cholangitis, microgallbadder, gallbladder dyskinesia, and symptomatic cholelithiasis have been reported with a wide range of prevalence ${ }^{14}$. The factors responsible for this phenotypic variability are not known. Moreover, the pathophysiology of liver disease remains uncertain and so far, no therapy has proven effective to prevent the progression of CFLD $4,13,15$. A full understanding of the pathophysiology and the effect of environmental and non-CFTR genetic influences in the context of CFLD development would help improve management and develop new drug therapies.

This review provides the latest knowledge in the pathophysiology of CFLD and CF genetic modifier research in CFLD.

\section{Pathophysiology of CFLD: what is known?}

CFTR expression in the hepatobiliary system, is restricted to the apical membrane of cholangiocytes lining the intra- and extrahepatic bile ducts. CFTR drives fluid and bicarbonate secretion in cholangiocytes ${ }^{16,17}$. It has been assumed that the importance of CFTR in maintaining homeostasis in the biliary tree was related to the "bicarbonate umbrella" hypothesis, in which a proper alkaline balance is critical to prevent cholangiocyte injury by hydrophobic bile acids ${ }^{18,19}$. According to this hypothesis, the loss of CFTR function would contribute to $\mathrm{pH}$ dysregulation and thereby would promote cholangiocyte injury ${ }^{13}$. Evidence has also been provided to indicate that the loss of CFTR affected innate immunity and promoted gut dysbiosis, which in turn would induce intestinal inflammation, endotoxinemia and CFLD 20-24. New functions of CFTR in cholangiocytes were recently described, i.e., the regulation of 
cell polarity, and of toll-like receptor 4 (TLR4) response via the activation of the tyrosine kinase Src ${ }^{20}$. This latter study showed that in cholangiocytes, CFTR promoted the assembly of a protein complex that maintained Src in an inactive state ${ }^{20}$. Thus, in the biliary epithelium exposed to endotoxins, when CFTR is defective, this complex does not assemble, resulting in the self-activation of Src, and subsequent activation of TLR4 and of nuclear factor kappa-lightchain-enhancer of activated $\mathrm{B}$ cells $(\mathrm{NF}-\mathrm{\kappa B})$. This mechanism may contribute to an increase in the production of proinflammatory cytokines leading to peribiliary inflammation and fibrosis ${ }^{20}$. Moreover, the impairment in barrier function is expected to increase the back-diffusion of toxic bile acids, and thereby to further aggravate peribiliary inflammation and fibrogenesis. We alo recently showed in CF mouse models, that the development of CFLD features required the combination of CFTR defect with both genetic and environmental factors such as diet-induced dysbiosis 24,25 .

\section{Molecular mechanisms underlying phenotypic differences in CFLD}

As an approach to unravel the molecular mechanisms underlying phenotypic differences in CFLD, investigators sought to determine if the presence and/or severity of CFLD was associated with a transcriptomic signature. Pereira et al. provided evidence for a transcriptional basis of CFLD pathogenesis ${ }^{26}$. The liver biopsies of children with CFLD compared with those of children with biliary atresia and normal controls displayed differences in the expression of multiple genes related to tissue remodeling and fibrogenesis including chemokines, collagens and matrix metalloproteases. In CFLD, regardless of fibrosis severity, compared with normal controls and biliary atresia, the expression of genes involved in tissue remodeling was decreased, notably the expression of plasminogen activator inhibitor-1 (PAI-1, up to 25-fold) and of tissue inhibitor of metalloproteinase-1 (TIMP-1). By contrast, the expression of genes encoding matrix metalloproteases or involved in the immue response such as interleukins and 
their receptors were overexpressed in the liver of patients with CFLD ${ }^{26}$. PAI-1 and TIMP-1 are two key regulators of matrix remodeling. PAI-1, encoded by the gene SERPINE1, belongs to a family of serine protease inhibitors. It acts as an inhibitor of the tissue-type and urokinase-type plasminogen activators, which convert plasminogen into plasmin ${ }^{27}$, a protease required for the activation of matrix metalloproteases and soluble factors such as hepatocyte growth factor or transforming growth factor $\beta$. TIMP-1 is an inhibitor of matrix metalloproteases. Therefore, the decrease in the production of PAI-1 and TIMP-1 is expected to increase the activation of matrix metalloproteases and ultimately to increase matrix turnover. Of particular interest, another member of the same family of serine protease inhibitors, namely SERPINA1, has been identified as a modifier gene of CFLD ${ }^{28}$. Because the genes encoding PAI-1 and CFTR are both located on the long arm of chromosome 7 , future studies should also determine whether there is any genetic linkage between these two loci. Overall, transcriptomic analyses supported the role of cytokine and chemokine signaling pathways, hepatic stellate cell activation and matrix remodeling in the pathogenesis of CFLD, as suggested by several studies ${ }^{29-31}$.

We recently demonstrated that CFLD, i.e., cholangiopathy features, was markedly influenced by the genetic background in $C f t r$ knockout mice ${ }^{24}$. By comparing $C f t r$ knockout mice in a congenic C57BL/6J or mixed C57BL/6J;129/Ola background, we found that cholangiopathy occurred only in mice of the congenic background. Transcriptomic analyses of the liver showed that mice in the congenic versus mixed background, overexpressed genes conveying an hyperinflammatory state whereas genes related to innate and adaptative immunity were underexpressed, including the absence of CD1d2 expression, in the C57BL/6J background. Protein-protein interactions predicted by the analysis of genes overexpressed in the liver of the congenic mice formed a large network, displaying links between inflammation, fibrosis and cell (presumably cholangiocyte) proliferation ${ }^{24}$. This result supported the view that 
cholangiopathy arising in $\mathrm{CF}$, is the result of an ill-adapted innate immune response to endotoxins coming from the intestine and triggering a pro-inflammatory response ${ }^{20}$.

\section{Phenotype-genotype correlations}

Despite all efforts provided by investigators to elucidate the pathophysiology of CFLD, the reason why only one-third of patients with CF develop liver disease, and why the severity of CFLD is so highly variable remains poorly known.

There are currently more than 2,000 variations listed in the CFTR mutation databases (https://cftr2.org). Among all CFTR variations, the most prevalent one, accounting for approximately $70 \%$ of all $C F T R$ alleles worldwide, results in the deletion of a phenylalanine at position 508 (F508del). CFTR variations are categorized into six classes based on their functional consequences and subsequent residual activity at the apical membrane of epithelial cells. Class I, II, and III mutations (i.e., severe mutations) result in a complete loss of the chloride-channel function; in contrast, class IV, V, and VI mutations (i.e., mild mutations) are associated with altered conductance properties, reduced synthesis or defective stability of normal CFTR, compatible with residual CFTR membrane activity ${ }^{1}$.

CFLD is largely limited to pancreatic insufficient patients with biallelic loss-of-function mutations in CFTR (i.e., class I, II, or III mutations on both allele) ${ }^{4,10,12,32,33}$. Meconium ileus was also shown to be a risk factor for severe CF liver disease with portal hypertension 4, 7, 10, 12, 34, 35. There is also an increased prevalence of severe CFLD in males (2:1) over females, in keeping with the fact that male gender is a factor of fibrosis progression in all chronic liver diseases ${ }^{4,8}$.

Recently, we have confirmed in a large cohort of CF patients that the F508del homozygous CFTR genotype was a risk factor for the development of liver involvement and severe CFLD ${ }^{4}$. However, the heterogeneous phenotype of liver involvement in CF patients bearing the same 
CFTR genotype, including CF siblings, suggests that non-CFTR genetic variations, i.e., modifier genes contribute to the risk of severe CFLD ${ }^{36,37}$, and to the liver phenotypic heterogeneity, as shown for lung and intestinal phenotypes in $\mathrm{CF}^{3,38,39}$. A poor concordance of liver disease in sibling pairs with CF has been reported, which argues against a major role of environmental factors ${ }^{40}$.

\section{The CF genetic modifier research}

Initial searches for non-CFTR genetic variants that might influence liver phenotype in patients with CF, used a candidate gene approach, which is based on biological plausibility. However, this approach has not been conclusive so far, mainly because the understanding of the pathophysiology of CF liver disease was insufficient. Genes that have been studied as putative modifiers of CF liver disease include SERPINA1, encoding the alpha-1-antitrypsin protein, genes which variants cause hemochromatosis (HFE, TFR2 and FPN1), and genes encoding glutathione S-transferase P1 (GSTP1), mannose binding lectin 2 (MBL2), angiotensin I converting enzyme (ACE), transforming growth factor beta 1 (TGFB1), interleukin 8 (currently known as CXCL8), as well as ATP-binding cassette subfamily B member 4, which is defective in progressive familial intrahepatic cholestasis type 3, intrahepatic cholestasis of pregnancy and drug-induced cholestasis ${ }^{28,41-44}$. However, only SERPINA1 has been confirmed as a modifier of liver disease severity in CF. Most studies have investigated cohorts of small size and phenotyping did not address the development of severe (biliary) cirrhosis associated with portal hypertension. The largest two-stage control study of CFLD (defined as advanced liver disease with portal hypertension) focused on 9 variations in 5 candidate genes (SERPINA1, ACE, GSTP1, MBL2, and TGFB1) that had previously been screened in patients with various degree of CFLD severity $28,41,42,44$. Genetic analyses of the initial cohort (124 patients with CFLD and 843 control patients without CFLD) showed that a single copy of the SERPINA1 Z allele and 
each additional copy of the $T G F B 1$ codon $10 \mathrm{C}$ allele were associated with significantly increased odds of CFLD ${ }^{28}$. In the replication study including an additional 136 patients with CFLD and 1,088 without CFLD, the SERPINA1 Z allele was confirmed as a modifier of liver disease in $\mathrm{CF}$, but not the $T G F B 1$ codon 10 variant. Overall, this unique two-stage control study showed a strong association of the Z-allele of the $\alpha_{1}$-anti-protease (SERPINA1) gene with CFLD in a total of 260 patients with CFLD versus 1,931 patients with CF and no CFLD. Of particular interest with respect to pathophysiology, the alpha-1-antitrypsin variant protein accumulates not only in hepatocytes but also in cholangiocytes, in subjects bearing the SERPINA1 Z alleles ${ }^{45}$. One may speculate that the accumulation of alpha-1-antitrypsin variant induces an endoplasmic reticulum stress in cholangiocytes as it does in hepatocytes. This may aggravate endoplasmic reticulum stress induced by the accumulation of F508del CFTR and predispose cholangiocytes to injury of other causes, e.g. endotoxins or toxic bile.

While genome-wide association data have not yet been reported in CFLD, a different methodology was used in a recent work using a system biology approach combining database and literature mining, gene expression study, network analysis, pathway enrichment analysis and protein-protein interactions to examine functional relationships between reported genes ${ }^{46}$. This large study led to the identification of novel modifier genes potentially involved in CFLD: SLC33A1 (NCBI ID: 9197), GPNMB (NCBI ID: 10457), NCF2 (NCBI ID: 4688), RASGRP1 (NCBI ID: 10125), LGALS3 (NCBI ID: 3958) and PTPN13 (NCBI ID: 5783) ${ }^{46}$. Pathways associated with these genes highlighted the innate immune system and Ubiquitin $\mathrm{C}$ (UBC, OMIM: 191340) as the central node linking CF to liver disease ${ }^{46,47 .}$

In conclusion, CFLD is characterized by progressive damage of bile ducts with inflammation, and peribiliary fibrosis in portal tracts. There is increasing evidence to indicate that CFTR 
deficiency in the biliary epithelium induces an increased vulnerability to injury of different causes including bacterial endotoxin and a hyperinflammatory response of cholangiocytes or in their vicinity. The reason why, among CF patients with the same CFTR genoytype, a minority progress to multilobular cirrhosis in the first decade of life still remains poorly understood but genes involved in the innate immune system are candidate modifiers. A multifaceted approach combining genome-wide association studies, and gene expression studies will hopefully reveal significant gene modifiers for CF liver disease. Challenges for the future are to develop novel therapies to prevent or halt the progression of this disease.

\section{Key points}

- Cystic fibrosis (CF)-related liver disease (CFLD) manifests as focal biliary fibrosis in approximately one third of patients, but fibrosis may progress to multilobular cirrhosis by the end of the second decade of life in a minority of patients ( 5 to $10 \%$ ).

- The heterogeneous severity of liver involvement in patients bearing the same CFTR genotype, including CF siblings, suggests that non-CFTR genetic variations contribute to the risk of severe liver disease

- So far, the only modifier gene validated in CFLD is SERPINA1 (alpha-1-antitrypsin) Z allele

- Evidence has recently been provided to indicate that cholangiopathy arising in $\mathrm{CF}$, is the result of an ill-adapted innate immune response to endotoxins coming from the intestine and triggering a pro-inflammatory response

- Using a system biology approach, novel modifier genes potentially involved in CFLD were identified and associated pathways highlighted the innate immune system and Ubiquitin C (UBC, OMIM: 191340), as the central node linking CF to liver disease. 
- Genome-wide association data, not yet available, are urgently needed to identify potential targets for modulating liver disease severity in CF.

\section{References and recommended reading}

Papers of particular interest, published within the annual period of review, have been highlighted as:

* of special interest

** of outstanding interest

1. Rowe SM, Miller S, Sorscher EJ. Cystic fibrosis. N Engl J Med 2005;352:1992-2001. 2. Amaral MD. Novel personalized therapies for cystic fibrosis: treating the basic defect in all patients. J Intern Med 2015;277:155-66.

3. Corvol H, Blackman SM, Boelle PY, et al. Genome-wide association meta-analysis identifies five modifier loci of lung disease severity in cystic fibrosis. Nat Commun $2015 ; 6: 8382$.

4. Boelle PY, Debray D, Guillot L, et al. Cystic Fibrosis Liver Disease: Outcomes and Risk Factors in a Large Cohort of French Patients. Hepatology 2018.

* This large database study including 3,328 patients with CF describes the incidence of CFLD and the impact of ursodeoxycholic acid therapy on the incidence of severe CFLD.

5. Cystic Fibrosis Foundation Patient Registry ADRttCD, Bethesda, Maryland (p73). https://www.cff.org/2014-Annual-Data-Report.pdf n.d.).

6. Vawter GF, Shwachman H. Cystic fibrosis in adults: an autopsy study. Pathol Annu 1979;14 Pt 2:357-82.

7. Debray D, Lykavieris P, Gauthier F, et al. Outcome of cystic fibrosis-associated liver cirrhosis: management of portal hypertension. J Hepatol 1999;31:77-83.

8. Stonebraker JR, Ooi CY, Pace RG, et al. Features of Severe Liver Disease With Portal Hypertension in Patients With Cystic Fibrosis. Clin Gastroenterol Hepatol 2016;14:12071215 e3. 
9. Debray D, Kelly D, Houwen R, et al. Best practice guidance for the diagnosis and management of cystic fibrosis-associated liver disease. J Cyst Fibros 2011;10 Suppl 2:S29-36. 10. Colombo C, Battezzati PM, Crosignani A, et al. Liver disease in cystic fibrosis: A prospective study on incidence, risk factors, and outcome. Hepatology 2002;36:1374-82. 11. Lindblad A, Glaumann H, Strandvik B. Natural history of liver disease in cystic fibrosis. Hepatology 1999;30:1151-8.

12. Lamireau T, Monnereau S, Martin S, et al. Epidemiology of liver disease in cystic fibrosis: a longitudinal study. J Hepatol 2004;41:920-5.

13. Debray D, Narkewicz MR, Bodewes F, et al. Cystic Fibrosis-related Liver Disease: Research Challenges and Future Perspectives. J Pediatr Gastroenterol Nutr 2017;65:443-448.

*This review highlights gaps in current knowledge, and defines critical areas to address including changes in registry data collection, non-invasive monitoring of liver disease progression, and relevant disease mechanisms to optimise future therapeutic options.

14. Assis DN, Debray D. Gallbladder and bile duct disease in Cystic Fibrosis. J Cyst Fibros 2017;16 Suppl 2:S62-S69.

15. Cheng K, Ashby D, Smyth RL. Ursodeoxycholic acid for cystic fibrosis-related liver disease. Cochrane Database Syst Rev 2017;9:CD000222.

16. Cohn JA, Strong TV, Picciotto MR, et al. Localization of the cystic fibrosis transmembrane conductance regulator in human bile duct epithelial cells. Gastroenterology 1993;105:1857-64.

17. Chinet T, Fouassier L, Dray-Charier N, et al. Regulation of electrogenic anion secretion in normal and cystic fibrosis gallbladder mucosa. Hepatology 1999;29:5-13. 18. Beuers U, Hohenester S, de Buy Wenniger LJ, et al. The biliary $\mathrm{HCO}(3)(-)$ umbrella: a unifying hypothesis on pathogenetic and therapeutic aspects of fibrosing cholangiopathies. Hepatology 2010;52:1489-96.

19. Beuers U, Maroni L, Elferink RO. The biliary $\operatorname{HCO}(3)(-)$ umbrella: experimental evidence revisited. Curr Opin Gastroenterol 2012;28:253-7.

20. Fiorotto R, Villani A, Kourtidis A, et al. The cystic fibrosis transmembrane conductance regulator controls biliary epithelial inflammation and permeability by regulating Src tyrosine kinase activity. Hepatology 2016;64:2118-2134.

** This study reveals a novel function of CFTR as a regulator of toll-like receptor 4 responses and cell polarity in biliary epithelial cells.

21. Blanco PG, Zaman MM, Junaidi O, et al. Induction of colitis in cftr-/- mice results in bile duct injury. Am J Physiol Gastrointest Liver Physiol 2004;287:G491-6. 
22. Scirpo R, Fiorotto R, Villani A, et al. Stimulation of nuclear receptor peroxisome proliferator-activated receptor-gamma limits NF-kappaB-dependent inflammation in mouse cystic fibrosis biliary epithelium. Hepatology 2015;62:1551-62.

** This study unravels a novel function of PPAR-gamma in controlling biliary epithelium inflammation and suggest that impaired activation of PPAR-gamma contributes to the chronic inflammatory state of CFTR-defective cholangiocytes.

23. Harmon GS, Dumlao DS, Ng DT, et al. Pharmacological correction of a defect in PPAR-gamma signaling ameliorates disease severity in Cftr-deficient mice. Nat Med 2010;16:313-8.

24. Debray D, El Mourabit H, Merabtene F, et al. Diet-induced dysbiosis and genetic background synergize with cystic fibrosis transmembrane conductance regulator deficiency to promote cholangiopathy in mice. Hepatol Commun 2018; https://doi.org/10.1002/hep4.1266.

** This experimental study in CF mice provides evidence that CFTR deficiency causes abnormal intestinal permeability, which, combined with diet-induced dysbiosis and immune-related genetic susceptibility, promotes CF-related cholangiopathy.

25. Debray D, Rainteau D, Barbu V, et al. Defects in gallbladder emptying and bile Acid homeostasis in mice with cystic fibrosis transmembrane conductance regulator deficiencies. Gastroenterology 2012;142:1581-91 e6.

26. Pereira TN, Lewindon PJ, Greer RM, et al. Transcriptional basis for hepatic fibrosis in cystic fibrosis-associated liver disease. J Pediatr Gastroenterol Nutr 2012;54:328-35.

27. Kruithof EK. Plasminogen activator inhibitors--a review. Enzyme 1988;40:113-21.

28. Bartlett JR, Friedman KJ, Ling SC, et al. Genetic modifiers of liver disease in cystic fibrosis. Jama 2009;302:1076-83.

29. Ramm GA, Shepherd RW, Hoskins AC, et al. Fibrogenesis in pediatric cholestatic liver disease: role of taurocholate and hepatocyte-derived monocyte chemotaxis protein-1 in hepatic stellate cell recruitment. Hepatology 2009;49:533-44.

30. Lewindon PJ, Pereira TN, Hoskins AC, et al. The role of hepatic stellate cells and transforming growth factor-beta(1) in cystic fibrosis liver disease. Am J Pathol 2002;160:1705-15.

31. Pereira TN, Lewindon PJ, Smith JL, et al. Serum markers of hepatic fibrogenesis in cystic fibrosis liver disease. J Hepatol 2004;41:576-83.

32. Colombo C, Apostolo MG, Ferrari M, et al. Analysis of risk factors for the development of liver disease associated with cystic fibrosis. J Pediatr 1994;124:393-9. 
33. Duthie A, Doherty DG, Williams C, et al. Genotype analysis for delta F508, G551D and R553X mutations in children and young adults with cystic fibrosis with and without chronic liver disease. Hepatology 1992;15:660-4.

34. Maurage C, Lenaerts C, Weber A, et al. Meconium ileus and its equivalent as a risk factor for the development of cirrhosis: an autopsy study in cystic fibrosis. J Pediatr Gastroenterol Nutr 1989;9:17-20.

35. Chryssostalis A, Hubert D, Coste J, et al. Liver disease in adult patients with cystic fibrosis: a frequent and independent prognostic factor associated with death or lung transplantation. J Hepatol 2011;55:1377-82.

36. Gallati S. Disease-modifying genes and monogenic disorders: experience in cystic fibrosis. Appl Clin Genet 2014;7:133-46.

37. Salvatore F, Scudiero O, Castaldo G. Genotype-phenotype correlation in cystic fibrosis: the role of modifier genes. Am J Med Genet 2002;111:88-95.

38. Drumm ML, Konstan MW, Schluchter MD, et al. Genetic modifiers of lung disease in cystic fibrosis. N Engl J Med 2005;353:1443-53.

39. Blackman SM, Deering-Brose R, McWilliams R, et al. Relative contribution of genetic and nongenetic modifiers to intestinal obstruction in cystic fibrosis. Gastroenterology 2006;131:1030-9.

40. Castaldo G, Fuccio A, Salvatore D, et al. Liver expression in cystic fibrosis could be modulated by genetic factors different from the cystic fibrosis transmembrane regulator genotype. Am J Med Genet 2001;98:294-7.

41. Henrion-Caude A, Flamant C, Roussey M, et al. Liver disease in pediatric patients with cystic fibrosis is associated with glutathione S-transferase P1 polymorphism. Hepatology 2002;36:913-7.

42. Gabolde M, Hubert D, Guilloud-Bataille M, et al. The mannose binding lectin gene influences the severity of chronic liver disease in cystic fibrosis. J Med Genet 2001;38:310-1. 43. Tomaiuolo R, Degiorgio D, Coviello DA, et al. An MBL2 haplotype and ABCB4 variants modulate the risk of liver disease in cystic fibrosis patients: a multicentre study. Dig Liver Dis 2009;41:817-22.

44. Arkwright PD, Pravica V, Geraghty PJ, et al. End-organ dysfunction in cystic fibrosis: association with angiotensin I converting enzyme and cytokine gene polymorphisms. Am J Respir Crit Care Med 2003;167:384-9.

45. Callea F, Fevery J, Massi G, et al. Storage of alpha-1-antitrypsin in intrahepatic bile duct cells in alpha-1-antitrypsin deficiency (Pi Z phenotype). Histopathology 1985;9:99-108. 
46. Trouve P, Genin E, Ferec C. In silico search for modifier genes associated with pancreatic and liver disease in Cystic Fibrosis. PLoS One 2017;12:e0173822.

** This unique study using a system biological approach identified novel modifier genes potentially involved in CFLD.

47. Cui J, Chen Y, Wang HY, et al. Mechanisms and pathways of innate immune activation and regulation in health and cancer. Hum Vaccin Immunother 2014;10:3270-85. 